\title{
Identifying Muscle Strength Imbalances in Athletes using Motion Analysis Incorporated with Sensory Inputs
}

\author{
Sameera S. Vithanage ${ }^{1}$, Maneesha S. Ratnadiwakara ${ }^{2}$, \\ Damitha Sandaruwan ${ }^{3}$, Shiromi Arunathileka ${ }^{4}$, \\ Maheshya Weerasinghe ${ }^{5}$ \\ School of Computing, University of Colombo \\ Colombo 07, Sri Lanka
}

\author{
Chathuranga Ranasinghe ${ }^{6}$ \\ Allied Health Sciences Unit \\ Faculty of Medicine \\ University of Colombo \\ Colombo 07, Sri Lanka
}

\begin{abstract}
Movement analysis is one of the commonly used methods in the context of physiotherapy to identify dysfunctions in the human musculoskeletal system. The overhead squat is a popular movement pattern that is also approved by NASM (National Academy of Sports Medicine of USA) among the various movement patterns that are used to identify muscle dysfunctions. It is commonly used to draw conclusions on an athlete's muscle imbalance in the clinical field based on observed compensations of the movement pattern. It is used by trainers as well as fitness enthusiasts to routinely assess their movement patterns. The correct execution of movements in every athlete is crucial since the incorrect bio-mechanics can result in injuries that would take a considerable amount of time to recover through rehabilitation. Thus, there is a need to evaluate injury risks accurately. The primary purpose of this research is to propose a method of detecting muscle imbalances in collegiate athletes with the aid of a low-cost motion tracking device. This proposed method facilitates the detection of muscle imbalances in both upper-body as well as lower-body during the execution of the overhead squat.
\end{abstract}

Keywords-Musculoskeletal imbalance; movement analysis; motion tracking; injury prevention

\section{INTRODUCTION}

Movement analysis or movement screening is commonly used to assess the biomechanics of the human body and identify individuals with high injury risk [1]. There are numerous movement screening methods that exist to identify movement quality and muscle dysfunctions. Functional Movement Screen (FMS) and Movement Competency Screen (MCS) are a couple of such tools approved by the National Academy of Sports Medicine, USA (NASM) [2]. The overhead squat is used in both of the aforementioned screening tools, as one of its components making it an overall movement quality indicator. NASM focuses more on the compensations surrounding each joint involved in the movement pattern and the possible overactive and under-active muscle groups contributing to these compensations [2] which provides an in-depth understanding of the functionality of the musculoskeletal system. These screening methods are widely used in the clinical field considering its ease of implementation because it only requires observations and domain knowledge to evaluate muscle quality. Through these observational data, clinicians can identify abnormal movement patterns or dysfunctions. According to Bishop et al. [2], these movement anomalies are said to represent the muscle imbalances caused by inflexibility, muscle weakness, and unbalanced muscle activation.

The previously mentioned clinical evaluation methods hold several inconveniences when it comes to the scope of collegiate athletes. If the athletes are not routinely assessed, the dysfunctions in the musculoskeletal system can be identified only when pain or discomfort is present. Sports injuries can become critical and force athletes to refrain from their practices for rehabilitation purposes. Furthermore, those injuries can resurface in the future, unless proper recovery procedures are followed [3]. The overhead squat test is a movement pattern specifically used for the clinical identification of musculoskeletal imbalance [4]. When considering the assessment of movement dysfunctions, the overhead squat has a few advantages over the previously mentioned screening methods. The time consumed for the evaluation is considerably less than when performed multiple movements as in FMS. Based on this study [2], it also covers all the key joints in the kinetic chain and it is also a commonly used movement pattern in strength and conditioning context.

The primary motivation for this research study is to propose an ICT based solution for athletes to routinely assess the quality of their musculoskeletal system in terms of muscle imbalances, which affects the magnitude of their sports performances. The early identification of these imbalances greatly benefits in the process of preventing major common muscle injuries. The research study focuses on a non-invasive and costeffective solution. It also does not require a domain expert to carry out the assessment of the musculoskeletal system.

\section{RELATED WORK}

Several research studies have been done to detect muscle strength imbalance and asymmetry using a variety of sensors. One such study has been done to detect muscle imbalances by identifying abnormalities in the gait cycle [5]. A markerless motion capturing device was used to capture different phases in the gait cycle. Three graphs were generated to denote the variation of the ankle, knee and hip angles against time. The resulting graphs were compared against the standard gait cycle 
graph to detect a person with muscle imbalances. Despite the fact that the solution can be used to self-evaluate muscle imbalances, it does not detect muscle imbalances in the upper body and it can not help identify possible overactive and underactive muscle groups.

A previous study [6] was done to validate the reliability of the vertical jump force test(VJFT) in assessing strength asymmetry of athletes. A single force plate was used to measure the force exerted by each leg during the execution of the jump. One leg was placed on the force plate and the other on a level wooden platform. The reading from the force plate was compared against the results of the isokinetic leg extension test and the isometric leg press test in which the results have shown a strong correlation. This has validated the reliability of using the vertical jump force test for assessing bilateral strength asymmetry. However, the vertical jump force test does not allow the evaluation of different muscle groups in the lower limb but only considers the force exerted from the entire lower limb as a whole.

A similar study [7] was done to examine the bilateral differences in the ground reaction forces during the overhead deep squat test. A twin-force plate system was used to measure the peak ground reaction force during this test. The study was done on a sample of young soccer athletes and the results indicate that there appears to be a 'trigger point' during early adolescence that marks the increase of bilateral imbalance. The magnitude of imbalance increases as the players get older. The results suggest that early detection of bilateral imbalances and taking corrective measures is crucial in preventing musculoskeletal injuries.

According to Mauntel et al. [8], there can be biomechanical differences between males and females during the execution of the overhead squat. The particular study was done using an electromagnetic motion tracking system interfaced with a force platform to measure the lower extremity kinematics and kinetics during the descent phase of the squat. The results have indicated several differences between the males and females which concluded that gender-specific injury prevention programs should be developed.

Another study was done to compare the objective methods and manual (real-time) methods in grading the functional movement screen [9]. The study was done by comparing the FMS grades given by a certified FMS tester and those given by an objective inertial-based motion capture system. The inertial measurement unit sensors were placed in the subject's body and the readings obtained while executing the components of the FMS was used to score the subjects. According to Whiteside et al, manual evaluation of the FMS is susceptible to error and there lies a need to develop a standard procedure in grading FMS performance.

Based on the related works, only one research has been previously conducted to detect muscle imbalance using a motion sensor and it was limited to the lower body. Furthermore, there was no identification of potential overactive and under-active muscles. All the other aforementioned studies have used force plate systems, electromagnetic tracking systems and inertial movement sensors to detect human movement which cannot be considered as practical solutions to detect muscle imbalances regularly by athletes due to the high cost of the equipment
TABLE I. SUMMARY OF THE EVALUATION OF RELATED WORKS

\begin{tabular}{|c|c|c|c|c|c|}
\hline Related Work (Reference No.) & 5 & 6 & 7 & 8 & 9 \\
\hline Economical & Yes & No & No & No & No \\
\hline Non-Invasive & Yes & Yes & Yes & No & No \\
\hline Ease of Implementation & Yes & Yes & Yes & No & No \\
\hline Self-Evaluation & Yes & No & No & No & No \\
\hline Whole Body & No & No & No & No & Yes \\
\hline
\end{tabular}

[2]. The objective of this research study is to provide a solution that is cost-effective and can be used to identify muscle imbalances by oneself. The proposed solution can detect potential overactive and under-active muscles and also help to track the progress of the treatments while avoiding the errors caused when evaluating muscle imbalances manually. The summary of the above mentioned previous studies and the evaluation of them regarding various factors are stated in Table I.

\section{Methodology And Design}

At present, as mentioned in a previous study review [10], there are no previous studies that has been done using a motion tracking device to carry out the movement analysis in the context of detecting muscle imbalances in collegiate athletes. This study analyses how specific joint angles and joint distances differ when performing a movement test (Overhead Squat). One of the main aspects of this study is to develop generic overhead squat models for healthy athletes in order to identify individuals who are having muscle dysfunctions.

The overhead squat was selected as a suitable movement pattern considering several factors. In the context of collegiate athletes, the time is a limiting factor, thus using one movement pattern like the overhead squat that facilitates the detection of muscle imbalances in the entire body is more suitable than FMS which takes a longer time period for the assessment. Furthermore, it measures key joint measurements in the kinetic chain as mentioned by Bishop et al. [2].

\section{A. Subjects}

All the participants in this study were collegiate athletes from the University of Colombo, who're regular players in their respective sports. Overall, 40 athletes volunteered to participate including 23 sportsmen and 17 sportswomen aged between 20-25. All the subjects considered for the study were not reported having previous musculoskeletal injuries or having current injuries. Furthermore, the subjects had not undergone any rehabilitation treatments or self-reported treatments. At the moment of data collection, the subjects were in good physical condition without any discomfort or pain. The subjects were mainly categorized according to their gender since BMI values change accordingly. Those who professionally practice sports were also excluded from the sample since they might have developed specific movement patterns to increase their performance in respective sports, which cannot be considered as dysfunctional patterns.

\section{B. Procedure}

1) Data Collection: Orbbec Astra Pro, a device that is equipped with a depth sensor, was used to collect joint measurement data in this study. The joint positions were correctly 
identified using the input from the depth sensor and without the use of any on-body markers. Since the device does not affect the movement of the subjects, they performed the Overhead Squat as they naturally do in front of a physiotherapist or a clinician. Shoulder, Hip, Knee and Ankle joints positions in the 3D space were captured with the device in order to calculate the respective joint angles and distances.

2) Laboratory Setup: The data collection process was carried out in a laboratory environment to increase the accuracy of the results by reducing unnecessary noise. An indoor research facility was used as the setting to carry out the experimental tests. The windows in the room were covered so that the sunlight would not interfere with the infrared rays emitted from the sensor. The camera was placed at a range where it can capture the whole body movement of the subject, which was determined as 3 meters away from the device and 1 meter above the ground.

3) Training Protocol followed by subjects: Prior to the testing procedure, each subject was given the following instructions as to how to perform the overhead squat accurately as instructed by Dr. Chathuranga Ranasighe [4]. This protocol was followed in order to ensure that the subject understands the correct technique of performing the overhead squat; thus reducing anomalous readings caused due to incorrect technique rather than any existing muscle imbalances.

1) sit on a chair

2) place the feet shoulder-width apart

3) repeat 5-10 times

4) repeat 5-10 times

5) push from the heel when standing up

6) ask whether the subject feels the Gluteal (back) muscles engaging

7) sit while slightly touching the chair

8) ask to push with heels

9) observe knee movement

10) ask not to move the knee beyond toes

11) repeat 5 times

After successfully training the sample, the subjects were asked to perform the overhead squat without the chair and with the correct technique.

4) Data Preparation and Cleansing: In order to obtain accurate joint position measurements, the subjects were instructed to not wear very dark or black color clothing during the experiment. The windows in the laboratory environment were covered to avoid infrared rays in sunlight interfering with the same emitted by the depth sensor.

Multiple measures were taken to generalize the conditions for all the samples as much as possible. The data was collected during afternoon hours, from $1 \mathrm{pm}$ to $4 \mathrm{pm}$. The athlete was not exhausted or did not express any form of physical discomfort during the data collection period. The instructions were given to all the subjects in the sample by the same person (experienced with the training protocol) to avoid any misinterpretations in the training session. The observations were done with the guidance from a domain expert. The collected data were rescaled from 1-100 time frames using a python script to fit every subject on the same scale. Some data were excluded due to capturing errors in the device.

\section{Mathematical Modeling}

The X, Y, Z coordinates captured by the device can be used to obtain angle values using the below mentioned mathematical models.

1) Mathematical model to measure the distance between two joints.: Points $\mathrm{P}$ and $\mathrm{Q}$ are two points in the $3 \mathrm{D}$ space as in Fig. 1. The coordinates of point $\mathrm{P}$ is given by $\left(x_{1}, y_{1}, z_{1}\right)$ and point $\mathrm{Q}$ by $\left(x_{2}, y_{2}, z_{2}\right)$. Using the Pythagorean theorem, the distance between two points in a $2 \mathrm{D}$ plane is calculated by the equation:

$$
d(P, Q)=\sqrt{\left(x_{1}-x_{2}\right)^{2}+\left(y_{1}-y_{2}\right)^{2}}
$$

Similarly the distance between two point in the 3D space can be calculated by the following equation:

$$
d(P, Q)=\sqrt{\left(x_{1}-x_{2}\right)^{2}+\left(y_{1}-y_{2}\right)^{2}+\left(z_{1}-z_{2}\right)^{2}}
$$

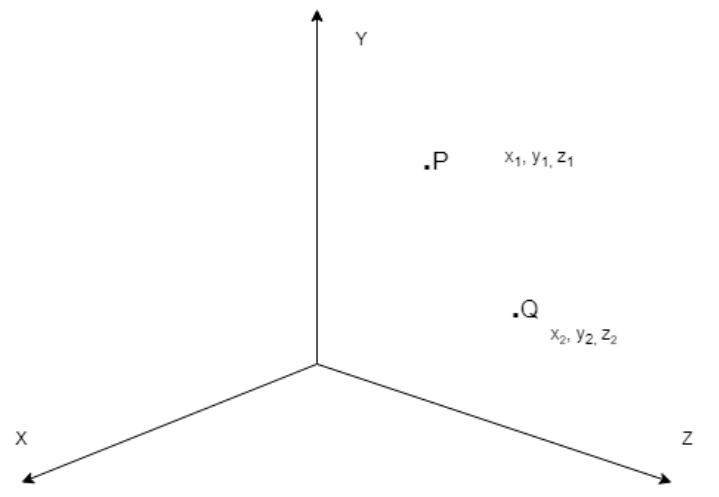

Fig. 1. Mathematical model for measuring joint distances

2) Mathematical Model to measure angles values: Consider PO (a) and QO (b) as two vectors that intersects in the $3 \mathrm{D}$ space shown in Fig. 2. The angle between the two vectors POQ is denoted by $\theta .|a|$ signifies the magnitude the of the vector PO (a) which is equal to:

$$
|a|=\sqrt{x_{1}^{2}+y_{1}^{2}+z_{1}^{2}} \rightarrow 1
$$

The dot product of the two vectors can be calculated using the following formula.

$$
a . b=x_{1} * x_{2}+y_{1} * y_{2}+z_{1} * z_{2} \rightarrow 2
$$

Angles between two lines in a $3 \mathrm{D}$ space is equal to the angle subtended by the two vectors which are parallel to those lines. The angle between the two vectors can be calculated from the following formula.

$$
\begin{gathered}
a . b=|a| *|b| * \cos (\theta) \\
\cos (\theta)=\frac{a . b}{|a| *|b|}
\end{gathered}
$$


Substituting from 1 and 2 ,

$$
\cos (\theta)=\frac{x_{1} * x_{2}+y_{1} * y_{2}+z_{1} * z_{2}}{\sqrt{x_{1}^{2}+y_{1}^{2}+z_{1}^{2}} * \sqrt{x_{2}^{2}+y_{2}^{2}+z_{2}^{2}}}
$$

Therefore;

$$
\theta=\cos ^{-1} \frac{x_{1} * x_{2}+y_{1} * y_{2}+z_{1} * z_{2}}{\sqrt{x_{1}^{2}+y_{1}^{2}+z_{1}^{2}} * \sqrt{x_{2}^{2}+y_{2}^{2}+z_{2}^{2}}}
$$

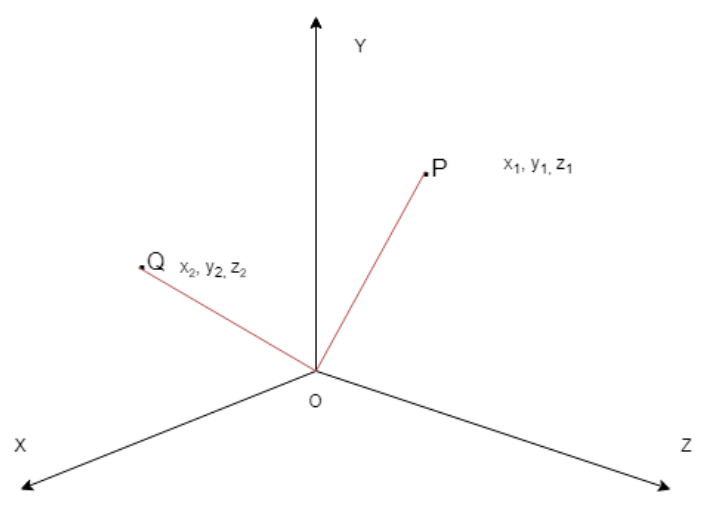

Fig. 2. Mathematical model for measuring joint angles

\section{Developing Models for Healthy Athletes}

A clinician observes a checklist of areas for compensations when the subject performs the overhead squat. The above mathematical models were used to represent the movement pattern of a subject when performing the overhead squat to identify these compensations. Based on the clinician's expert opinion, we developed the movement patterns corresponding to a healthy subject, in order to identify the imbalanced subjects.

1) Anterior view: Observations in this view mainly focused on knees and feet, depicted in Fig. 3. Compensations to look out for are the "toe-out", "knees move in" and "knees move out". In the correct anterior view, the hip, knee and ankle joints on either side of the body should be aligned. The joint distance between right and left knee joint positions were measured in the span of a single overhead squat (from standing position to squat position and back to standing position). The distance between right and left ankles was measured in the same way which was used to represent the movement of the toes. If there's a significantly high variance in distance (percentage difference), it was concluded that the subject was having a movement compensation.

Fig. 4 shows how the average distance between knees varies in the healthy male sample. Similarly, the female sample data also can be plotted to understand the variations as depicted in Fig. 5.

2) Lateral View: Lateral view observations depicted in Fig. 6, involve the lumbo-pelvic hip complex (LPHC) and upper body positions. Compensations often observed are excessive forward lean and arms falling forward. As described in the domain, the trunk should be parallel to the lower leg during the descent phase of the squat. If not, it can be concluded as excessive forward lean. The arms falling forward is observed

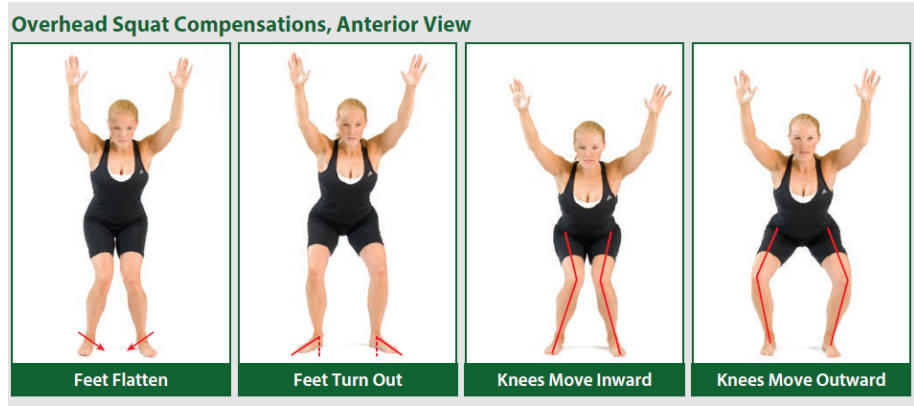

Fig. 3. Anterior view compensations [11]

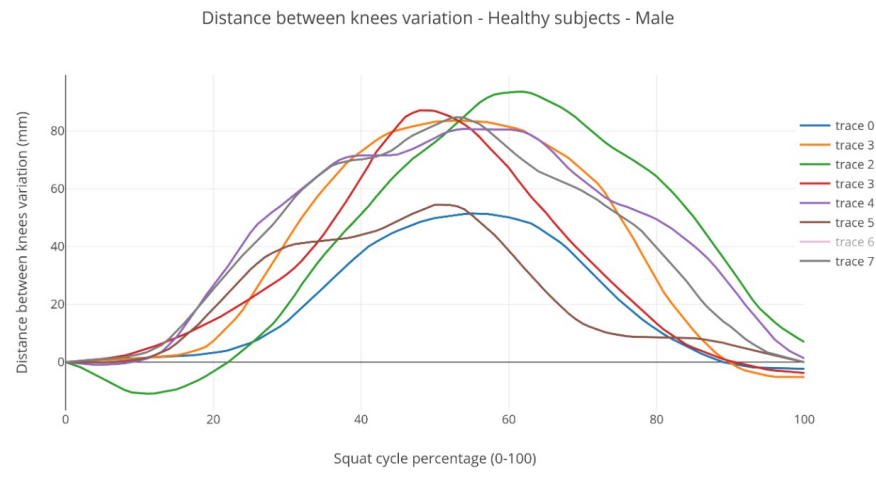

Fig. 4. The variation of knee distance - Male

when elbows are fully extended above head, elbow joint, shoulder and hip center should be aligned as a straight line. For the purpose of measuring this, the shoulder joint angle was calculated with respect to the hip joint and elbow joint. If there's a significantly high variance in the shoulder angle value, it was concluded that the subject was having a movement compensation.

3) Posterior View: Posterior view observations depicted in Fig. 7, include the areas of feet and lumbo-pelvic hip complex. The behavior of the feet when doing the overhead squat can be observed in this view. The feet should be touching the ground in the entirety of the squat. To observe the flattening of the medial longitudinal arch, the movement of the ankles from the floor during the squat was measured and whether the pattern is within a normal measurement range. For better accuracy, the subjects were asked to remove any footwear before performing the movement.

The graph in Fig. 8 shows how the ankle moves away from the floor plane. This can be taken as the observation of heel lift compensation. The sample of healthy athletes has shown little to no heel lift as observed from the data.

Similarly, it can be plotted for the healthy female sample as shown in Fig. 9.

The same process was used to generate graphs for the healthy sample of males and females with respect to each of the five compensation categories that are defined in the overhead squat movement pattern. These graphs with defined threshold values were taken as the standard for an average athlete doing 


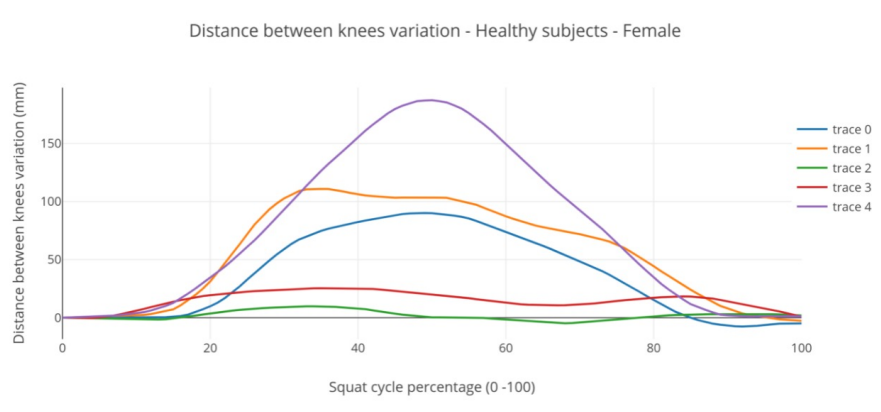

Fig. 5. The variation of knee distance - Female

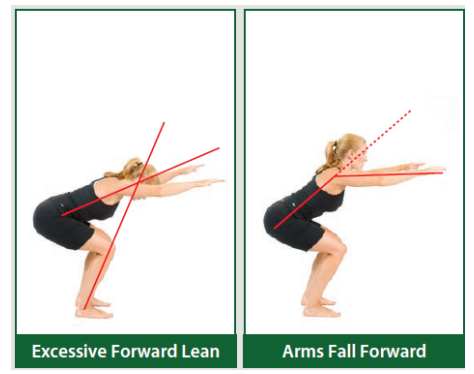

Fig. 6. Lateral view compensations [11]

the movement pattern of the overhead squat. The experiment was done using these graphs to evaluate a selected sample of males and females.

\section{E. High-Level Research Design}

The high-level research design illustrated in Fig. 10, consists of mainly two phases; Data collection and data analysis. In the data collection phase, the overhead squat was used as the specific movement pattern which was performed by the selected subjects in front of the sensor under the laboratory environment. Joint positions were captured with the device as $\mathrm{X}, \mathrm{Y}, \mathrm{Z}$ coordinates. These data were used to calculate the respective joint angles and distances with the aid of the previously described mathematical models. The above graphs were created to represent the movement pattern of the respective subject.

The next phase was the data analysis. The relative graphs generated from the Data collection phase were used as the input for the analysis phase. These graphs were compared against the respective acceptable movement threshold graphs that were previously created. If the values deviate from the defined threshold values of the acceptable graphs, it was identified as the subject having an imbalance. Using the specific deviated joint measurements, potential overactive and underactive muscle groups were identified. Finally, the status of the subjects was given.

\section{RESUlts}

As discussed in the previous section, there are mainly five compensations of the overhead squat that were focused on in this study. For each of these compensation categories, the collected data were mapped against the previously developed

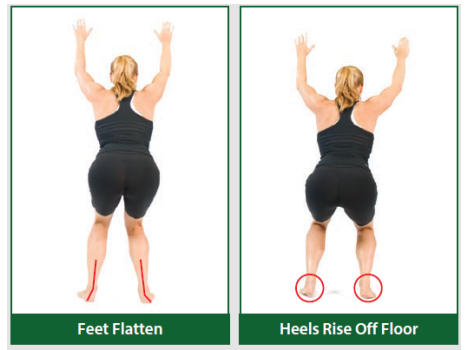

Fig. 7. Posterior view compensations [11]

Heel lift healthy subjects - male

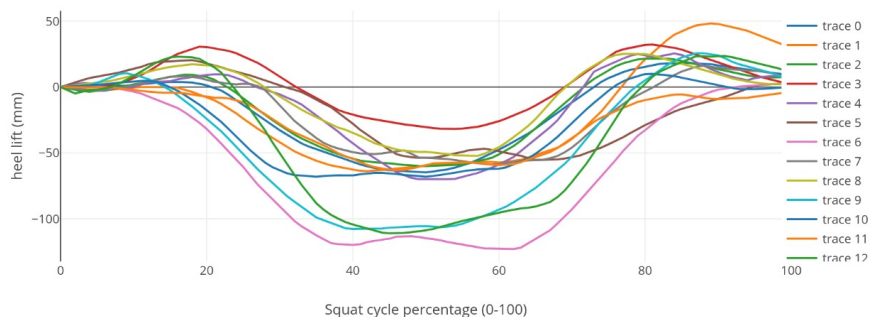

Fig. 8. The variation of heel from the floor plane - Male

healthy sample graphs. The evaluation results obtained from the system as imbalanced or not are compared with the evaluation results of a domain expert to conclude how accurate the system output is. In order to do that confusion matrices were created for each of these categories.

The graphs in Fig. 11 and Fig. 12 represent the models of min-max threshold graphs for Knee distance variation of males and females respectively. The graph in Fig. 13 represents the knee distance variation of a healthy male while the graph in Fig. 14 represents the same of a male with the compensation. Similarly, Fig. 15 and Fig. 16 depict the knee distance variation of a healthy female and a female with the compensation, respectively.

Graphs were created for each of the subjects under the above mentioned compensation categories to determine the presence or absence compensations in the subjects.

\section{A. Min and Max threshold graphs}

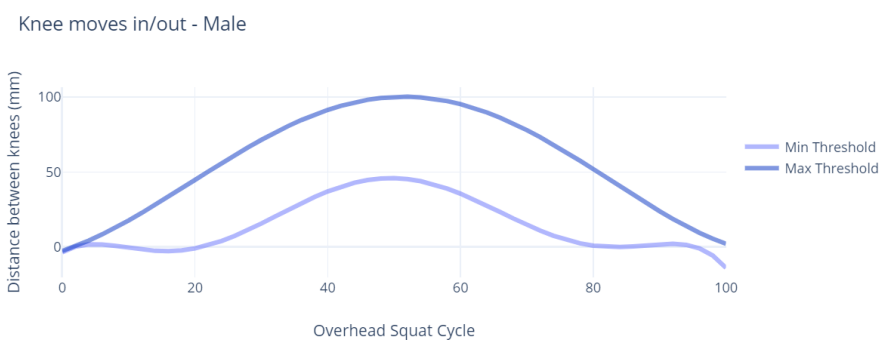

Fig. 11. Min and Max Threshold values for Knee distance - Male 


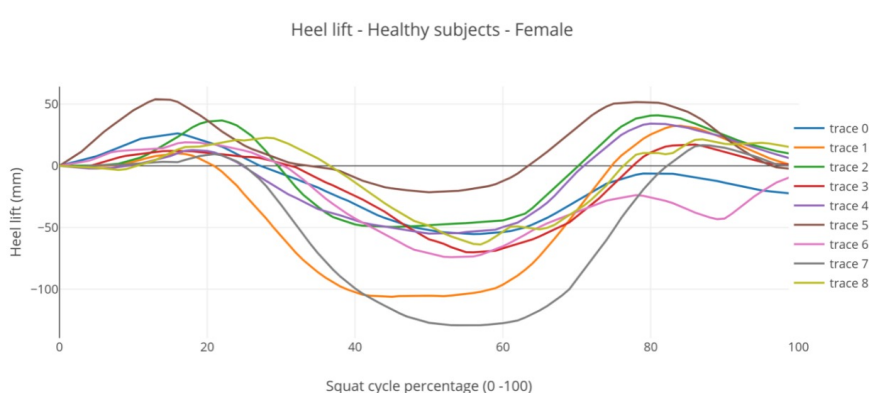

Fig. 9. The variation heel from floor plane - Male
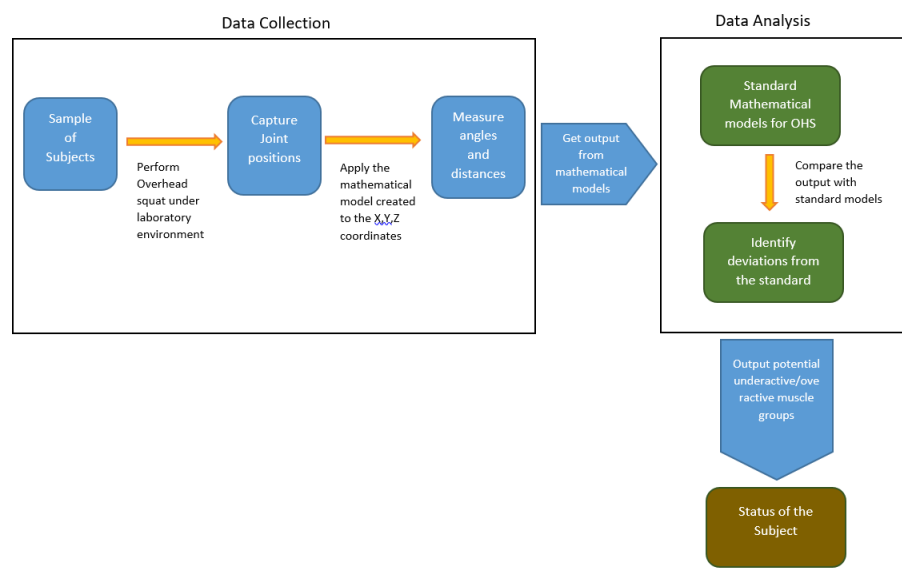

Fig. 10. Mathematical model for measuring joint angles

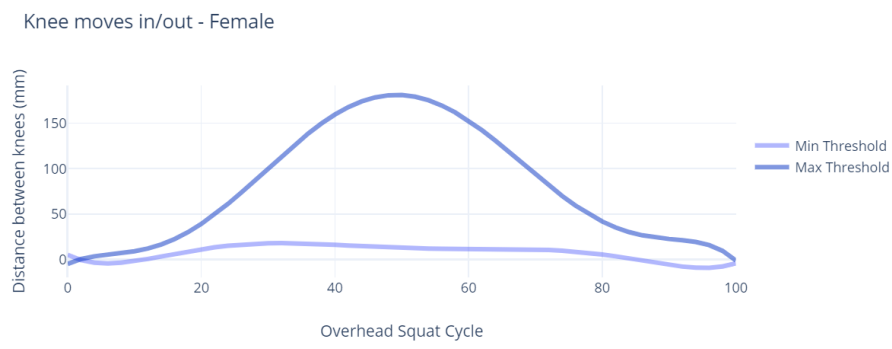

Fig. 12. Min and Max Threshold values for knee distance - Female

\section{B. Healthy and non-healthy graphs for Male}

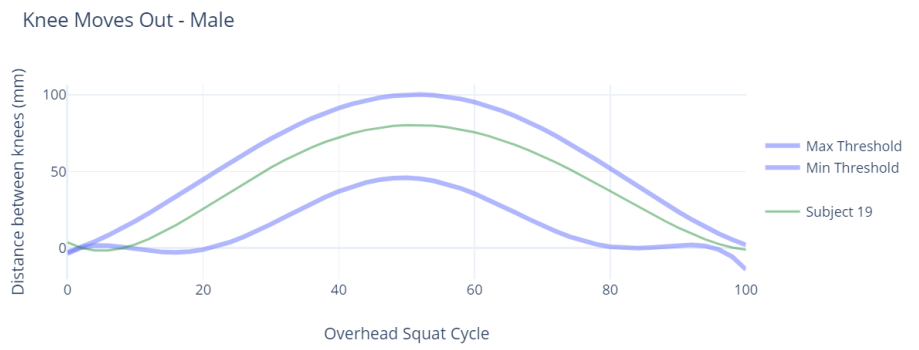

Fig. 13. Healthy subject graph for knee distance - Male

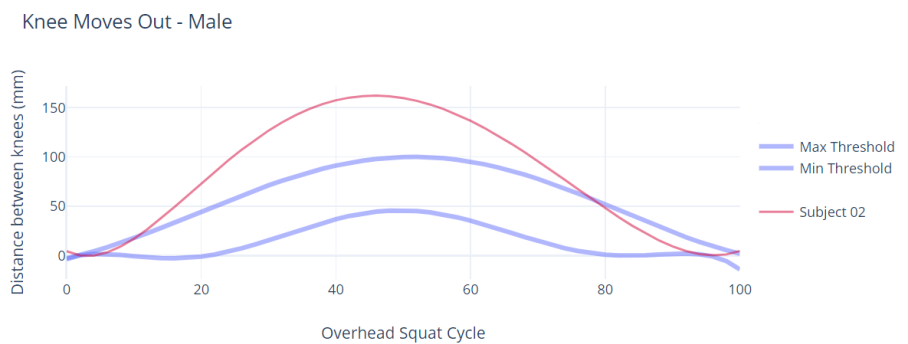

Fig. 14. Non-healthy subject graph for knee distance - Male

\section{Healthy and non-healthy graphs for Female}

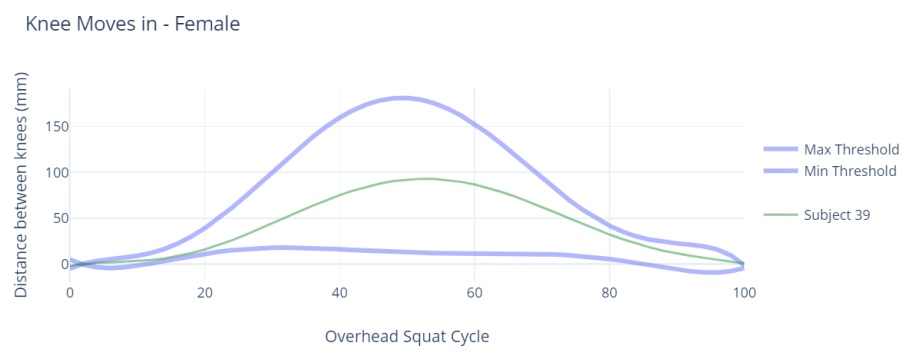

Fig. 15. Healthy subject graph for knee distance - Female

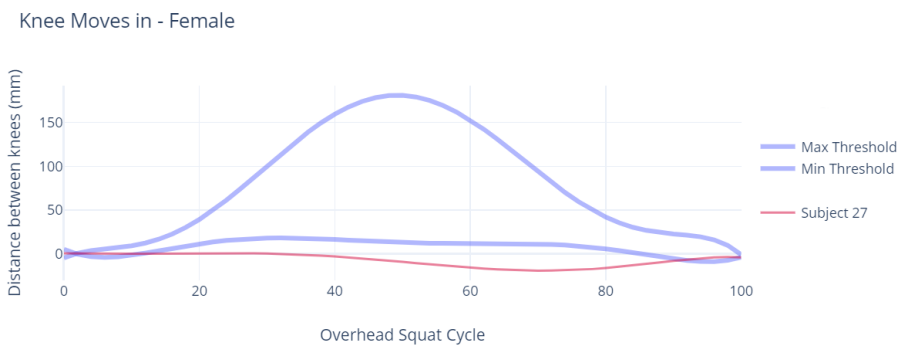

Fig. 16. Non-healthy subject graph for knee distance - Female

\section{DISCUSSION}

The purpose of this section is to address the various aspects of the obtained results in this research study. Based on the available literature, this is a novel approach to detect muscle imbalances in athletes using movement analysis. Overall 10 graphical models were created to represent the correct movement behavior pattern of the overhead squat of both male and female athletes. We have identified several deviations from the theoretical ideal pattern with the results obtained with respect to above mentioned five categories.

\section{A. Arms Falling Forward}

Ideally, the shoulder angle should be 180 degrees, though, from the results, it was observed that none of the athletes can reach that angle. The males have ranged starting around 170 degrees to 160 degrees. But the females' range is lower than the male range. It started from around 160 degrees to 140 degrees. The confusion matrix for this compensation is available in Table II. 
TABLE II. CONFUSION MATRIX - ARMS FALLING ForWARD

\begin{tabular}{|c|c|c|c|}
\cline { 2 - 4 } \multicolumn{1}{c|}{} & \multicolumn{3}{|c|}{ System Prediction } \\
\hline \multirow{4}{*}{$\begin{array}{c}\text { Domain Expert } \\
\text { Opinion }\end{array}$} & $\begin{array}{c}\text { Positive } \\
\text { (Imbalanced) }\end{array}$ & $\begin{array}{c}\text { Negative } \\
\text { (Healthy) }\end{array}$ \\
\cline { 2 - 4 } & $\begin{array}{c}\text { Positive } \\
\text { (Imbalanced) }\end{array}$ & $\mathrm{TP}=31$ & $\mathrm{FN}=2$ \\
\cline { 2 - 4 } & $\begin{array}{c}\text { Negative } \\
\text { (Healthy) }\end{array}$ & $\mathrm{FP}=0$ & $\mathrm{TN}=5$ \\
\hline
\end{tabular}

\section{B. Knee Valgus and Knee Varus}

As we observed the captured data, the distance between the left and right knee joints are varied from subject to subject. Normally females have less distance due to their small build. A specific behavior we identified from the calculation of knee distance is that the male subjects have shown the knee varus but not knee valgus compensation. Whereas in female subjects, the knee valgus compensation is commonly seen. It was also confirmed by the domain expert that behavior can be seen in males and females differently. Table III depicts the results.

TABLE III. Confusion MAtriX - KNeE VALgus AND KNeE varus

\begin{tabular}{|c|c|c|c|}
\cline { 2 - 4 } \multicolumn{1}{c|}{} & \multicolumn{3}{c|}{ System Prediction } \\
\hline \multirow{3}{*}{$\begin{array}{c}\text { Domain Expert } \\
\text { Opinion }\end{array}$} & $\begin{array}{c}\text { Positive } \\
\text { (Imbalanced) }\end{array}$ & $\begin{array}{c}\text { Negative } \\
\text { (Healthy) }\end{array}$ \\
\cline { 2 - 4 } & $\begin{array}{c}\text { Positive } \\
\text { (Imbalanced) }\end{array}$ & $\mathrm{TP}=12$ & $\mathrm{FN}=1$ \\
\cline { 2 - 4 } & $\begin{array}{c}\text { Negative } \\
\text { (Healthy) }\end{array}$ & $\mathrm{FP}=1$ & $\mathrm{TN}=17$ \\
\hline
\end{tabular}

\section{Excessive Forward Lean}

Forward lean is detected by comparing the knee angle and the hip angle while doing the overhead squat. As shown in the results, the angles need to be approximately equal to be able to be identified as healthy. As per the domain expert's instructions, the knees should not go beyond the level of the toes. Only the knee angle and hip angle measurements were used to determine if the torso is parallel to the tibia; hence, there can be instances where the knee goes beyond toes while the knee and hip angles are within the healthy range. Even though that movement does not contribute heavily to the detection of muscle imbalances, that can be concluded as a limitation in the research study. The confusion matrix results are in Table IV.

TABLE IV. CONFUSION MATRIX - EXCESSIVE Forward LEAN

\begin{tabular}{|c|c|c|c|}
\cline { 2 - 4 } \multicolumn{1}{c|}{} & \multicolumn{3}{|c|}{ System Prediction } \\
\hline \multirow{3}{*}{$\begin{array}{c}\text { Domain Expert } \\
\text { Opinion }\end{array}$} & $\begin{array}{c}\text { Positive } \\
\text { (Imbalanced) }\end{array}$ & $\begin{array}{c}\text { Negative } \\
\text { (Healthy) }\end{array}$ \\
\cline { 2 - 4 } & $\begin{array}{c}\text { Positive } \\
\text { (Imbalanced) }\end{array}$ & $\mathrm{TP}=21$ & $\mathrm{FN}=3$ \\
\cline { 2 - 4 } & $\begin{array}{c}\text { Negative } \\
\text { (Healthy) }\end{array}$ & $\mathrm{FP}=0$ & $\mathrm{TN}=11$ \\
\hline
\end{tabular}

\section{Ankles Moving In}

Similar to knee distance, the ankle distance is also varied from subject to subject. As observed in the results, ankles normally would not go outwards. It is seen moving inwards in every case where there's heel lift compensation is present as well. Table V shows the results of Ankles moving in test.
TABLE V. CONFUSION MATRIX - ANKLES MOVING IN

\begin{tabular}{|c|c|c|c|}
\cline { 2 - 4 } \multicolumn{1}{c|}{} & \multicolumn{3}{c|}{ System Prediction } \\
\hline \multirow{4}{*}{$\begin{array}{c}\text { Domain Expert } \\
\text { Opinion }\end{array}$} & $\begin{array}{c}\text { Positive } \\
\text { (Imbalanced) }\end{array}$ & $\begin{array}{c}\text { Negative } \\
\text { (Healthy) }\end{array}$ \\
\cline { 2 - 4 } & $\begin{array}{c}\text { Positive } \\
\text { (Imbalanced) }\end{array}$ & $\mathrm{TP}=6$ & $\mathrm{FN}=5$ \\
\cline { 2 - 4 } & $\begin{array}{c}\text { Negative } \\
\text { (Healthy) }\end{array}$ & $\mathrm{FP}=0$ & $\mathrm{TN}=23$ \\
\hline
\end{tabular}

\section{E. Heel Lift}

Ideally, the heels should not be moved when performing the overhead squat. But the data gathered through the device shows that the joint position fluctuates slightly off the ground. This behavior is detected in all the evaluated subjects, thus we can conclude that can be caused by device error or environmental factors. If the deviation is large compared to others as discussed in the results chapter, it can be concluded that the subject shows a heel lift compensation. The final results are shown in Table VI.

TABLE VI. CONFUSION MATriX - HeEl LifT

\begin{tabular}{|c|c|c|c|}
\cline { 2 - 4 } \multicolumn{1}{c|}{} & \multicolumn{3}{c|}{ System Prediction } \\
\hline \multirow{3}{*}{$\begin{array}{c}\text { Domain Expert } \\
\text { Opinion }\end{array}$} & $\begin{array}{c}\text { Positive } \\
\text { (Imbalanced) }\end{array}$ & $\begin{array}{c}\text { Negative } \\
\text { (Healthy) }\end{array}$ \\
\cline { 2 - 4 } & $\begin{array}{c}\text { Positive } \\
\text { (Imbalanced) }\end{array}$ & $\mathrm{TP}=19$ & $\mathrm{FN}=1$ \\
\cline { 2 - 4 } & $\begin{array}{c}\text { Negative } \\
\text { (Healthy) }\end{array}$ & $\mathrm{FP}=0$ & $\mathrm{TN}=16$ \\
\hline
\end{tabular}

\section{F. Limitations and Constraints}

- The research study was done based on collegiate athletes which are limited to the sportsmen and sportswomen aged between 20 to 25 . However, the proposed solution can be applied to those who are pursuing general fitness goals as well.

- The overhead squat - the movement pattern used in the study, is specifically used in the clinical field to identify muscle imbalances. It is a basic functional movement that has been incorporated into many complex movement patterns in sports. The model for healthy athletes are generalized in this study, thus it cannot be applied for professional athletes who may have developed specific behavior patterns to increase performance in their respective sports.

- One of the constraints in the device is that the Orbbec Astra uses infrared rays to detect the joint positions. Thus, the subjects should not wear any black clothing as it affects the device's ability to detect the join positions accurately.

- Another device constraint is that the capturing of the joint positions should be done in a closed environment to avoid sunlight. The infrared rays from the sun can interfere with the infrared rays emitted from the device, causing it to give erroneous results.

\section{CONCLUSION}

Prevention of injuries is a widely discussed topic in the field of sports science. An abrupt injury once occurred, might 
restrict an athlete from engaging in routine practices or even crucial competitions in addition to the rehabilitation treatments and the costs associated. An injury might be decisive of an athlete's sports career as there lies a possibility of the same repeating which may prevent the subject from reaching his/her full potential with respect to the particular sport. Therefore, a mechanism to identify athletes with potential overactive and underactive muscles which may cause biomechanical disadvantages and injuries, in the long run, is needed.

This research study was focused on identifying imbalances in the musculoskeletal system using a motion capturing device in order to provide a cost-effective solution for athletes to self evaluate the condition of their musculoskeletal systems. However, there are several limitations to this study which may be potential future directions that this study can be expanded to. The research study was done focused on collegiate athletes who are not involved in sports at a professional level. Future studies can be conducted involving professional athletes by taking into account the specific movement patterns that are involved with each sport which may cause certain changes in the musculoskeletal system of such professional athletes.

Furthermore, the current research study was done based on mathematical models to determine the status of a subject. There is a possibility to conduct this research using machine learning techniques instead of mathematical models. However, there should be a sufficient dataset for the machine learning models to work with.

Currently, there are many wearable devices that athletes can use while engaged in sports without any discomfort such as fitness bands and foot reaction pressure detecting shoes. There is a possibility of incorporating the readings from such fitness devices to further improve the accuracy of this solution.

Also, the current solution considers each of the compensations of the overhead squat independently of each other. There were no previous studies done to determine the relationship between each of the compensations to observe how one compensation affects the other. Therefore, it possible to carry out future studies to determine the relationships between these compensations.

\section{REFERENCES}

[1] D. R. Bell, B. J. Vesci, L. J. DiStefano, K. M. Guskiewicz, C. J. Hirth, and D. A. Padua, "Muscle activity and flexibility in individuals with medial knee displacement during the overhead squat," Athletic Training \& Sports Health Care, vol. 4, no. 3, pp. 117-125, 2011.

[2] C. Bishop, M. Edwards, and A. Turner, "Screening movement dysfunctions using the overhead squat," 102016.

[3] J.-L. Croisier, B. Forthomme, M.-H. Namurois, M. Vanderthommen, and J.-M. Crielaard, "Hamstring muscle strain recurrence and strength performance disorders," The American Journal of Sports Medicine, vol. 30, no. 2, pp. 199-203, 2002.

[4] C. Ranasinghe, "Identifying muscle imbalances clinically," 2018.

[5] H. Tennakoon, C. Paranamana, M. Weerasinghe, D. Sandaruwan, and $\mathrm{K}$. Mahindaratne, "A novel musculoskeletal imbalance identification mechanism for lower body analyzing gait cycle by motion tracking," International Journal of Information Technology and Computer Science, vol. 10, no. 3, pp. 27-34, 2018.

[6] F. M. IMPELLIZZERI, E. RAMPININI, N. MAFFIULETTI, and S. M. MARCORA, "A vertical jump force test for assessing bilateral strength asymmetry in athletes," Medicine \& Science in Sports \& Exercise, vol. 39, no. 11, pp. 2044-2050, 2007.

[7] S. J. Atkins, I. Bentley, H. T. Hurst, J. K. Sinclair, and C. Hesketh, "The presence of bilateral imbalance of the lower limbs in elite youth soccer players of different ages," Journal of Strength and Conditioning Research, vol. 30, no. 4, pp. 1007-1013, 2016.

[8] T. C. Mauntel, E. G. Post, D. A. Padua, and D. R. Bell, "Sex differences during an overhead squat assessment," Journal of Applied Biomechanics, vol. 31, no. 4, pp. 244-249, 2015.

[9] D. Whiteside, J. M. Deneweth, M. A. Pohorence, B. Sandoval, J. R. Russell, S. G. McLean, R. F. Zernicke, and G. C. Goulet, "Grading the functional movement screen," Journal of Strength and Conditioning Research, vol. 30, no. 4, pp. 924-933, 2016.

[10] S. Vithanage, M. Ratnadiwakara, D. Sandaruwan, S. Arunathileka, M. Weerasinghe, and C. Ranasinghe, "A novel approach to detect muscle strength imbalances via motion analysis using sensory inputs," European Journal of Computer Science and Information Technology, vol. 46 , no. 5, pp. 37-50, 2018.

[11] T. Academy, "The overhead squat assessment," 2018. [Online]. Available: http://www.thefitnesstraineracademy.org/blog/the-overheadsquat-assessment/ 